\title{
Posterior Analysis of Sequential Normal Testing Procedure
}

\author{
Birjesh kumar \\ J.P Institute of Engg \\ \&Technology,Meerut \\ A-115 ganga sagar colony \\ Ganganagar,Meerut
}

\author{
Abha Chandra, $\mathrm{PhD}$ \\ Deptt. of Statistics \\ Meerut college,Meerut
}

\author{
K.K.Sharma, $\mathrm{PhD}$ \\ Retd Prof\&Head \\ Deptt.of Statistics \\ C.C.S University,Meerut
}

\begin{abstract}
Several studies deals with the non- robust character of various types of acceptance plans. Many such studies also analyze the robust character of sequential testing procedures when the underlying failure time distribution has a monotone failure rate. A vast literature on the life testing plans in the Bayesian framework is also available where updating prior with experimental data has been the main concern. Highlighting the point that the basic normal lifetime distribution can also be updated in respect of prior variations in the involve parameters. The present study deals with the analysis of the robust character of sequential normal testing procedures when the mean of the basic normal distribution is considered as a random variable. The robust character of the consistency of the random variable $n$, in view of prior variations, is also analyzed
\end{abstract}

\section{Keywords}

Robustness, SNTP, OC, ASN and Coefficient of variation function(C.V).

\section{INTRODUCTION}

An important contribution in the area of sequential test of statistical hypothesis is due to A. Wald (1947), who developed sequential probability ratio test (SPRT) for testing a simple hypothesis against a simple alternative. He obtained the expressions for operating characteristic (O.C.) and average sample numbers (ASN) function of the SPRT. The robustness of the SPRT, when the distribution under consideration has undergone a change, has been studied by various authors while dealing with various probability models, like Montagne and Singapurwala (1985), Sharma K.K. and Rana (1990), Sharma K.K. and Bhutani (1992) and others. The robustness of these procedures has been investigated in respect of producer's and consumer's risk and the ASN.

Further on using different life time models, the studies in [2A] include a vast literature on the life testing plans in Bayesian framework in which updating the prior with experimental data has been the main concern. However, in the Bayesian framework, it should be recognized that priors do have an impact on the basic distribution and therefore the basic distribution can be updated in view of prior variations. This updated basic distribution be used in the analysis. In the present paper we consider SPRT for testing the $H_{0}: \mu=\mu_{0}$ v/s $H_{1}: \mu=\mu_{1}$ when samples are $N\left(\mu, \sigma^{2}\right), \sigma^{2}$ being known. Further, when we consider $\mu$ to be a random variable we use the prior of $\mu$ to update the basic distribution, $N\left(\mu, \sigma^{2}\right)$ and use this basic updated distribution to study the robust character of SPRT when $\mu$ is considered to be a random variable. The concept has been highlighted in the present study.

Further, it was also noted that the basic distribution can be further updated in respect of the posterior distribution. This further updated distribution is also named as predictive basic distribution.

In view of above, the present paper considers the robust character of SPRT, when predictive distribution is used in the analysis. A comparison of OC, ASN curve and C.V curve in the corresponding situation has been used as the basis of analysis.

\section{NOTATIONS}

\begin{tabular}{|c|c|c|}
\hline$N(\mu, \sigma 2)$ & : & $\begin{array}{l}\text { Normal distribution with mean } \mu \text { and } \\
\text { variance } \sigma 2\end{array}$ \\
\hline p.d.f. & : & Probability density function \\
\hline SPRT & : & Sequential probability ratio test \\
\hline SNTP & : & Sequential normal testing procedure \\
\hline $\mathrm{L}(\mu)$ & : & $\begin{array}{l}\text { The OC function, the probability of } \\
\text { accepting HO when } \mu \text { is the true } \\
\text { parametric value }\end{array}$ \\
\hline $\mathrm{n}$ & : & $\begin{array}{l}\text { The sample size needed to terminate the } \\
\text { sequential testing. Thus } \mathrm{n} \text { is a random } \\
\text { variable. }\end{array}$ \\
\hline $\mathrm{E} \mu(\mathrm{n})$ & : & ASN functions for fixed $\mu$. \\
\hline $\mathrm{V} \mu(\mathrm{n})$ & : & $\begin{array}{l}\text { Variance of the random variable } \mathrm{n} \text { for } \\
\text { fixed } \mu\end{array}$ \\
\hline $\mathrm{CV} \mu(\mathrm{n})$ & : & $\begin{array}{l}\text { Co-efficient of variation of the random } \\
\text { variable } n \text { for fixed } \mu\end{array}$ \\
\hline$\alpha$ & $:$ & $\begin{array}{l}\text { Size of the type I error, also called } \\
\text { producer's risk in quality control } \\
\text { terminology. }\end{array}$ \\
\hline$\beta$ & : & $\begin{array}{l}\text { Size of the type II error, also called } \\
\text { consumer's risk in quality control } \\
\text { terminology. }\end{array}$ \\
\hline MTSF & . & Mean Time to system failure \\
\hline
\end{tabular}

\section{STATISTICAL BACKGROUND}

For developing the procedure we assume that (i)The failure

$$
\text { time distribution of } \mathrm{x} \text { is } N\left(\mu, \sigma_{1}^{2}\right) \text { with,p.d }
$$


$f_{1}\left(x, \mu, \sigma_{1}^{2}\right)=\frac{1}{\sigma_{1} \sqrt{2 \pi}} e^{-\frac{1}{2}\left(\frac{x-\mu}{\sigma_{1}}\right)^{2}} ;-\infty<x<\infty$

Here,$\mu$ is the MTSF and $\sigma 1$ is assumed known.

(ii) The lot to lot quality, $\mu$ is a random variable having its known conjugate prior distribution as normal with p.d.f.

$g\left(\mu, \theta, \sigma_{2}^{2}\right)=\frac{1}{\sigma_{2} \sqrt{2 \pi}} e^{-\frac{1}{2}\left(\frac{\mu-\theta}{\sigma_{2}}\right)^{2}} ;-\infty<\mu<\infty$,

(iii) Further let $\underset{\sim}{x}=\left(x_{1}, x_{2}, \ldots \ldots \ldots \ldots, x_{n}\right)$ be random sample of size $\mathrm{n}$ from the population in (1) then the posterior distribution of $\mu$ forgiven $\mathrm{X} \sim$ will be

$$
\begin{aligned}
& \pi(\underset{\sim}{\underline{\mu}})=\frac{\sqrt{n \sigma_{2}^{2}+\sigma_{1}^{2}}}{\sigma_{1}^{2} \sigma_{2}^{2}} \frac{1}{\sqrt{2 \pi}} e^{-\frac{1}{2}\left[\mu-\frac{n \bar{x} \sigma_{2}^{2}+\theta \sigma_{1}^{2}}{\sigma_{1}^{2}+n \sigma_{2}^{2}}\right] /\left(\frac{\sigma_{1}^{2}+n \sigma_{2}^{2}}{\sigma_{1}^{2} \sigma_{2}^{2}}\right)} \\
& \Longrightarrow \pi\left(\frac{\mu}{\underline{x}}\right) \square \pi\left(\lambda, \xi^{2}\right)
\end{aligned}
$$

where

$$
\lambda=\frac{n \bar{x} \sigma_{2}^{2}+\theta \sigma_{1}^{2}}{n \sigma_{2}^{2}+\sigma_{1}^{2}} \quad \xi^{2}=\frac{\sigma_{1}^{2} \sigma_{2}^{2}}{n \sigma_{2}^{2}+\sigma_{1}^{2}}
$$

(iv) Finally, in view of (1) and (3) the predictive distribution of the future observations [8] that is the updated compound distribution of $\mathrm{X}$ can also be obtained as

$$
\begin{aligned}
& h(x / \underset{\sim}{x})=\int_{-\infty}^{\infty} f\left(x, \mu, \sigma_{1}^{2}\right) \prod(\mu / \underset{\sim}{x}) d \mu \\
& \Rightarrow \frac{1}{\sqrt{2 \pi}\left(\sqrt{\xi^{2}+\sigma_{1}^{2}}\right)} e^{-\frac{1}{2}\left[\frac{x-\lambda}{\sqrt{\xi^{2}+\sigma_{1}^{2}}}\right]^{2}} \\
& \Rightarrow h(x / \underset{\sim}{x}) \square N\left(\lambda, \beta^{2}\right)=f_{3}\left(x, \lambda, \beta^{2}\right)
\end{aligned}
$$

Now here

$$
\begin{aligned}
& \mathrm{E}(\mathrm{X})=\left[\lambda=\frac{n \bar{x} \sigma_{2}^{2}+\theta \sigma_{1}^{2}}{n \sigma_{2}^{2}+\sigma_{1}^{2}}\right] \text { and } \\
& \operatorname{Var}(\mathrm{X})=\left[\beta^{2}=\sigma_{1}^{2}+\xi^{2}\right]
\end{aligned}
$$

Also the distribution of $\mathrm{x}$ in (4) is $\boldsymbol{N}\left(\boldsymbol{\lambda}, \boldsymbol{\beta}^{2}\right)$.The respective basic and predictive distributions in (1) and (4) $\sigma_{1}^{2}$ enæole us to analyse the robust character of SNTP with respect to $\alpha, \beta$ and $\mathrm{E} \mu(\mathrm{n})$ in the following situations-

(1) The basic distribution in (1) is used in the analysis when $\mu$ is considered as a constant.

(2) The predictive basic distribution in (4) which is updated in respect of posterior distribution of $\mu$ is used in the analysis when $\mu$ is considered as a random variable. Obviously, this updated distribution also includes information on experimental data.

\section{$\sigma_{4}^{2}>$ \&C AND ASN FUNCTIONS FOR SNTP WHEN $\mu$ IS CONSIDERED AS A CONSTANT}

In this case, SNTP for testing the null hypothesis $H_{0}: \mu=\mu_{0}=\lambda_{0}$ v/s $\quad H_{1}: \mu=\mu_{1}=\lambda_{1} \quad$ we assume that the sample observations are being sequentially recorded from the basic distribution in (1) i.e. $N\left(\mu, \sigma_{1}^{2}\right), \sigma_{1}^{2}$ being known.

Here, following [13]. The approximations to the OC function of the present SNTP can be obtained by considering the values of $g=g(\mu) \neq 0$ which satisfy

$$
E\left[\frac{f_{1}\left(x, \mu_{1}, \sigma_{1}\right)}{f_{1}\left(x, \mu_{0}, \sigma_{1}\right)}\right]^{g}=1
$$

$\int_{-\infty}^{\infty}\left[\frac{f_{1}\left(x, \mu_{1}, \sigma_{1}\right)}{f_{1}\left(x, \mu_{0}, \sigma_{1}\right)}\right]^{g} f_{1}\left(x, \mu, \sigma_{1}\right) d x=1$

On simplifying (5), one gets the following pair of parametric equations providing approximations to the OC function---

$$
\begin{gathered}
{\left[g=\frac{\left(\mu_{0}+\mu_{1}\right)-2 \mu}{\left(\mu_{1}-\mu_{0}\right)}\right]} \\
{\left[L(\mu)=\frac{A^{g}-1}{A^{g}-B^{g}}\right]}
\end{gathered}
$$

and

A plot of $L(\mu)$ against $\mu$ provides us the OC curve. Here,

$$
\begin{aligned}
& A=\frac{1-\beta}{\alpha}, \quad B=\frac{\beta}{1-\alpha} \\
& \mathcal{L}_{1} \neq \boldsymbol{\mu}_{\mathrm{O}}
\end{aligned}
$$

and

Further, the approximated formula for ASN of present SNTP will be

...(4)

$$
E_{\mu}(n)=\frac{L(\mu) \log B+(1-L(\mu)) \log A}{E_{\mu}(z)}
$$




$$
E_{\mu}(n)=\frac{L(\mu) \log B+(1-L(\mu)) \log A}{\frac{1}{2 \sigma_{1}^{2}}\left[\left(\mu_{0}^{2}-\mu_{1}^{2}\right)+2 \mu\left(\mu_{1}-\mu_{0}\right)\right]}
$$

Here

$$
E_{\mu}(z)=\frac{1}{2 \sigma_{1}^{2}}\left[\left(\mu_{0}^{2}-\mu_{1}^{2}\right)+2 \mu\left(\mu_{1}-\mu_{0}\right)\right]
$$

The notable features of the equation in (6) is that this is independent of $\sigma_{1}^{2}$.

Thus, the OC curves for the present SNTP remains static with variations in $\sigma_{1}^{2}$. As a consequence the points $\left[\mu_{0},(1-\alpha)\right]$ and $\left(\mu_{1}, \beta\right)$ on the OC curve also remain static with variations in $\sigma_{1}^{2}$ as such. The present SNTP is found to be robust in respect to ' $\alpha$ ' and ' $\beta$ ' with variation in $\sigma_{1}^{2}$. On the other hand, the expression for $E_{\mu}(n)$ in (7) is a function of $\sigma_{1}^{2}$. However, it seems logical that variation in $\sigma_{1}^{2}$ should obviously have an effect on the size of two errors, but the present SNTP is observed to be an exception in this regard. Still, the results in (6) and (7) provide us a basis for investigating the robustness of SNTP in respect of $\alpha, \beta$ and ASN when $\mu$ is considered as a random variable.

\section{ROBUSTNESS OF THE PRESENT SNTP IN RESPECT OF PRIOR VARIATIONS WITH EXPERIMENTAL DATA}

Given a sequence of observation $x_{1}, x_{2}, \ldots \ldots \ldots, x_{n}$ from (1) Suppose one wishes to test the null hypothesis

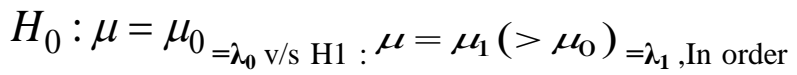
to study the robustness of the SPRT developed in section 4 in respect of OC and ASN function. We consider h1 as solution of the equation

$$
\begin{aligned}
& E\left[\int_{-\infty}^{\infty}\left\{\frac{f_{3}\left(x, \lambda_{1}, \beta\right)}{f_{3}\left(x, \lambda_{0}, \beta\right)}\right\}\right]^{h^{1}}=1 \\
& \int_{-\infty}^{\infty}\left[\frac{f_{3}\left(x, \lambda_{1}, \beta\right)}{f_{3}\left(x, \lambda_{0}, \beta\right)}\right]^{h^{1}} f_{3}(x, \lambda, \beta) d x=1
\end{aligned}
$$

it is notable that the expression in (9) is in respect of the predictive distribution in (3). The OC curve for testing $H_{0}: \mu=\mu_{0}=\lambda_{0} \quad$ v/s $\quad H_{1}: \mu=\mu_{1}=\lambda_{1} \quad$ is obtained approximately by the following pair of equations on solving (9), we get

$$
\begin{aligned}
& {\left[h^{\prime}=\frac{\left(\lambda_{1}+\lambda_{0}\right)-2 \lambda}{\left(\lambda_{1}-\lambda_{0}\right)}\right]} \\
& L(\lambda)=\frac{A^{h^{\prime}}-1}{A^{h^{\prime}}-B^{h^{\prime}}}
\end{aligned}
$$

Thus a comparison of the OC curve in (5) and (10) help us in analyzing the robust character of SNTP in respect of $\alpha$ and $\beta$ when updated prior has been used on using experimental data.

(3) Similarly, for analyzing the robustness of ASN function for SNTP we obtain

$$
\begin{aligned}
& E_{\lambda}(z)=E\left[\log \frac{f_{3}\left(x, \lambda_{1}, \beta\right)}{f_{3}\left(x, \lambda_{0}, \beta\right)}\right] \\
& =\int_{-\infty}^{\infty}\left(\log \frac{f_{3}\left(x, \lambda_{1}, \beta\right)}{f_{3}\left(x, \lambda_{0}, \beta\right)}\right) f_{3}(x, \lambda, \beta) d x \\
& \Rightarrow E_{\lambda}(z)=\frac{1}{2 \beta^{2}}\left[\left(\lambda_{0}^{2}-\lambda_{1}^{2}\right)+2 \lambda\left(\lambda_{1}-\lambda_{0}\right)\right]
\end{aligned}
$$

Here, also the expectation is taken in respect of the predictive normal distribution on using (9) one easily gets,

$$
E_{\lambda}(n)=\frac{L(\lambda) \log B+(1-L(\lambda)) \log A}{\frac{1}{2 \beta^{2}}\left[\left(\lambda_{0}^{2}-\lambda_{1}^{2}\right)+2 \lambda\left(\lambda_{1}-\lambda_{0}\right)\right]}
$$

For $\sigma 1=\beta$, Thus a comparison of the ASN in (7) and in (12) help us in analyzing the robust character of SNTP in respect of ASN when the prior can be updated in the form of posterior on using experimental data in Bayesian framework

\section{ANALYZING THE ROBUSTNESS OF THE CONSISTENCY OF N WHEN $\mu$ IS CONSIDERED AS A RANDOM VARIABLE.}

Recognizing the fact that in all sequential testing procedures $\mathrm{n}$ is a random variable, as such, the ASN function, i.e., $E_{\mu}(n)$ alone cannot be taken as a measure of effectiveness of the testing procedure. In this regard, $V_{\mu}(n)$

is a measure of the consistent behavior which can be analyzed by using a relative measure of dispersion, called the co-efficient of variation (C.V.) the C.V. in the present case will be

$$
C . V_{\mu}(n)=\frac{\sqrt{V_{\mu}(n)}}{E_{\mu}(n)} \times 100
$$

For developing formula for $\boldsymbol{C} \cdot \boldsymbol{V}_{\mu}(\boldsymbol{n})$ we proceed to develop an approximate formula for $V_{\mu}(n)$ as under. 
Following the assumptions as used in the derivation of $E_{\theta}(n)$ by Wald (1947), we consider

$\left(Z_{1}+Z_{2}+\ldots . Z_{N}\right)=\left(Z_{1}+Z_{2}+\ldots . Z_{n}\right)+\left(Z_{n+1}+\ldots . Z_{N}\right)$

Where

$$
Z_{\alpha}=\left[\log \frac{f_{2}\left(x_{\alpha}, \theta_{1}, \sigma^{2}\right)}{f_{2}\left(x_{\alpha}, \theta_{0}, \sigma^{2}\right)}\right]
$$

Also for $\alpha>n$, the random variable $Z_{\alpha}$ is distributed independently of $\mathrm{n}$.

Thus

$V\left(Z_{1}+Z_{2}+\ldots . Z_{N}\right)=V\left(Z_{1}+Z_{2}+\ldots . . Z_{n}\right)+V\left(Z_{n+1}+\ldots . . Z_{N}\right)$

Now, consider the term

$$
\begin{aligned}
& V\left(\sum_{i=n+1}^{N} Z_{i}\right)=V\left(\sum_{i=n+1}^{N} \frac{Z_{i}}{n}\right) \\
& =V\left[E\left(\sum_{i=n+1}^{N} \frac{Z_{i}}{n}\right)\right]+E\left[V\left(\sum_{i=n+1}^{N} \frac{Z_{i}}{n}\right)\right] \\
& =V[(N-n) E(z)]+E[(N-n) V(z)] \\
& =[E(z)]^{2} V(n)+V(z) E(N-n) \\
& =[E(z)]^{2} V(n)+V(z)[N-E(n)]
\end{aligned}
$$

On using (14) in (13), One gets

$$
V\left(\sum_{i=1}^{n} Z_{i}\right)+[E(z)]^{2} V(n)-V(z) E(n)=0
$$$$
V(n)=\frac{V(z) E(n)-V\left(\sum_{i=1}^{n} Z_{i}\right)}{[E(z)]^{2}}
$$

Since all the terms in $V(n)$ are function of $\mu$, thus the above can be re-written as

$$
V_{\mu}(n)=\frac{V_{\mu}(z) E_{\mu}(n)-V_{\mu}\left(\sum_{i=1}^{n} Z_{i}\right)}{\left[E_{\mu}(z)\right]^{2}}
$$

\section{6(a) $C . V_{\mu}(n)$ When $\mu$ is Constant}

In this case, the expression for $E_{\mu}(n)$ and $E_{\mu}(z)$ are as given in (7) and (8) respectively, further, it is easy to obtain

$$
\begin{aligned}
V_{\mu}(z) & =E_{\mu}\left(z^{2}\right)-\left[E_{\mu}(z)\right]^{2} \\
& =\frac{\left(\mu_{1}-\mu_{0}\right)^{2}}{\sigma_{1}^{2}}
\end{aligned}
$$

and

$$
V\left(\sum_{i=1}^{n} Z_{i}\right)=L(\mu)[1-L(\mu)][\log B-\log A]^{2}
$$

On using (7), (8) (17) and (18) in (16), one gets $V_{\mu}(n)$ in the present case and therefore,

$$
C V_{\mu}(n)=\frac{\sqrt{V_{\mu}(n)}}{E_{\mu}(n)} \times 100
$$

\section{6 (b) $C . V \cdot \lambda(n)$ when $\mu$ is random variable}

In this case, the expression for $E_{\lambda}(n)$ and $E_{\lambda}(z)$ are as given in (12) and (11) respectively, further one easily gets

$$
V_{\lambda}(z)=E_{\lambda}\left(z^{2}\right)-\left[E_{\lambda}(z)\right]^{2}=\frac{\left(\lambda_{1}-\lambda_{0}\right)^{2}}{\beta^{2}}
$$

and

$V\left(\sum_{i=1}^{n} z_{i}\right)=L(\lambda)[1-L(\lambda)]\{\log B-\log A\}^{2}$

On using (11), (12), (20) and (21) in (16) one easily get $V_{\lambda}(n)$ and consequently

$$
C \cdot V_{\lambda}(n)=\frac{\sqrt{V_{\lambda}(n)}}{E_{\lambda}(n)} \times 100
$$

Now, the trend in $C \cdot V_{\lambda}(n)$ and $C \cdot V_{\mu}(n)$ for varying $\lambda$ and $\mu$ can be used to study the robust character of the consistency of the random variable $n$, when $\mu$ is random variable.

\section{DISCUSSION}

Here it should be recognized that the basic distribution can be updated in view of prior variations in its parameter(s).Further, on using the experimental data, the prior can be updated in the form of posterior in the Bayesian framework. Still further, the basic distribution can be compounded with the posterior distribution to provide the updated compound or predictive normal distribution. In the process, we get the two basic normal distribution of $X$ in (1) and (4) representing two specific situations. As is evident from the analysis presented in section 4 , this predictive normal distribution enables us to 
study the robust characters of SNTP in respect of $\alpha, \beta$ and ASN function when prior has been updated with posterior as its distribution. For meeting this objective, we compare the generalized form of OC. curve in (6) with its particular form given in (10). Similarly, the generalized form of ASN in (7) is compared with its particular form in (12) so the last $C . V_{\mu}(n)$ and $C . V_{\lambda}(n)$

as given in (19) and (22) respectively are compared to analysis the robust character of the consistency of the random variable $\mathrm{n}$ when $\mu$ is considered as a random variable with posterior as its distribution. For clarity, we consider an example

\section{An example}

We have considered the SPRT for testing the hypothesis $H_{0}: \mu=\mu_{0}=\lambda_{0}=50 \quad$ v/s $\quad H_{1}: \mu=\mu_{1}=\lambda_{1}=47$ when observations are sequential recorded from $N\left(50, \sigma_{1}^{2}=64\right)$ and that for $\mu$ in (2) will be $N\left(50, \sigma_{2}^{2}=36\right)$

further suppose that the process sample information $X_{1}, X_{2}, \ldots \ldots X_{n}$ yields $\bar{X}=53$ for $\mathrm{n}=25$, Then on using (3) and (4), we have

$\lambda=\frac{n \bar{x} \sigma_{2}^{2}+\theta \sigma_{1}^{2}}{\sigma_{1}^{2}+n \sigma_{2}^{2}}=52.80$

$\left[\beta^{2}=\sigma_{1}^{2}+\xi^{2}\right]$

$\beta^{2}=66.39$

In other words, the predictive normal distribution of $\mathrm{X}$ $N(52.80,66.39)$. Now on using the expression in (6), (7) and (19), a few respective point on $L(\mu), E_{\mu}(n)$ and $C \cdot V_{\mu}(n)$ for varying $\mu$ have been summarized in Table-1. Now on considering the predictive basic distribution of $X$ in (4), the expression in (10), (12) and (22) the respective points on $L(\lambda), E_{\lambda}(n)$ and

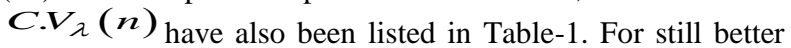
analysis of the non- robust character of SNTP in respect of $\alpha$, $\beta$ and ASN, when posterior prior has been used, the points in Table-1 are used to draw the curves for $L(\mu), L(\lambda)$, $E_{\mu}(n), E_{\lambda}(n)$ and $C \cdot V_{\mu}(n), C \cdot V_{\lambda}(n)$ for varying $\mu=E(\mu)=\lambda$ in figure 1,2 and 3 respectively.

\section{Analysis}

According to A. Wald [1947], the basis of selecting a desirable sequential test involves the following steps.

1. We first consider admissible sequential test of the same strength $(\alpha, \beta)$.

2. Then from among the admissible test, A test provide minimum $E_{\mu}(n)$, for all values of $\mu$ is considered as most desirable.

Table 1: Showing values of $L(\mu) / L(\lambda)$, $E_{\mu}(n) / E_{\lambda}(n)$ and $C . V \cdot \mu(n) / C \cdot V \cdot \lambda(n)$

for varying $\mu=E(\mu)=\lambda$

\begin{tabular}{|l|r|r|r|r|r|l|}
\hline $\begin{array}{c}\mu=\lambda= \\
\Theta\end{array}$ & $L(\mu)$ & $L(\lambda)$ & $E_{\mu}(n)$ & $E_{\lambda}(n)$ & $C V_{\mu}(n)$ & $C V_{\lambda}(n)$ \\
\hline 46.5 & 0.021 & 0.021 & 13.4975 & 13.5544 & 101.267 & 110.940 \\
\hline 47 & 0.049 & 0.049 & 16.7531 & 18.0739 & 119.832 & 120.056 \\
\hline 47.25 & 0.079 & 0.079 & 18.7012 & 19.0636 & 131.789 & 135.315 \\
\hline 47.5 & 0.125 & 0.125 & 21.086 & 21.2277 & 152.380 & 155.000 \\
\hline 47.75 & 0.187 & 0.187 & 23.0164 & 23.6206 & 196.423 & 182.621 \\
\hline 48 & 0.275 & 0.275 & 24.907 & 25.4733 & 253.045 & 259.476 \\
\hline 48.25 & 0.384 & 0.384 & 26.2772 & 26.2774 & 481.000 & 483.316 \\
\hline 48.75 & 0.615 & 0.615 & 26.0507 & 26.0509 & 477.233 & 480.000 \\
\hline 49 & 0.725 & 0.725 & 24.7938 & 25.4733 & 252.126 & 259.476 \\
\hline 49.25 & 0.813 & 0.813 & 22.9412 & 23.6206 & 184.769 & 190.512 \\
\hline 49.5 & 0.874 & 0.874 & 20.8313 & 21.1711 & 150.885 & 154.376 \\
\hline 49.75 & 0.92 & 0.92 & 18.7012 & 19.0183 & 131.578 & 135.000 \\
\hline 50 & 0.949 & 0.949 & 16.7531 & 16.9419 & 116.167 & 122.000 \\
\hline 50.5 & 0.978 & 0.978 & 13.4977 & 13.5261 & 101.015 & 102.767 \\
\hline & & & & & & \\
\hline
\end{tabular}




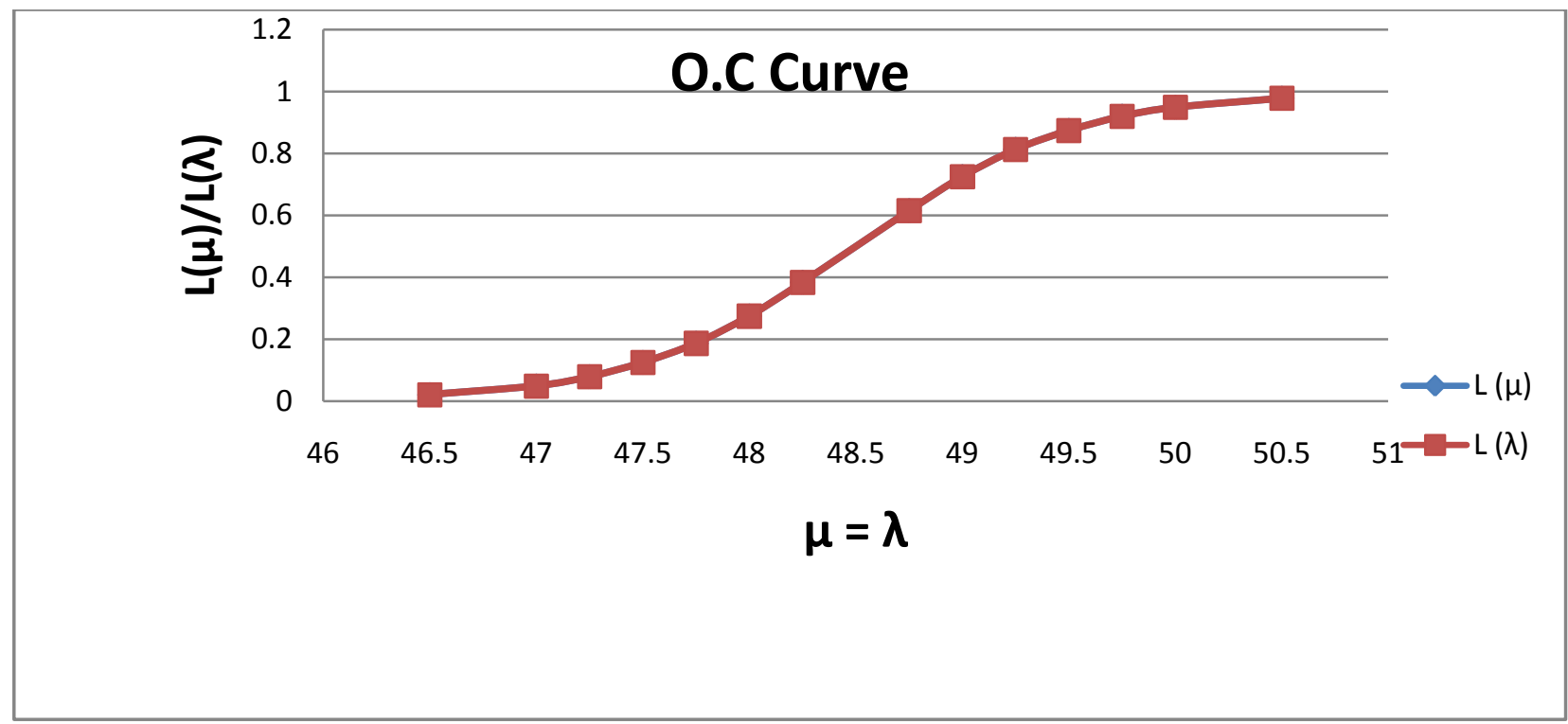

Fig:1 OC Curve for SNTP

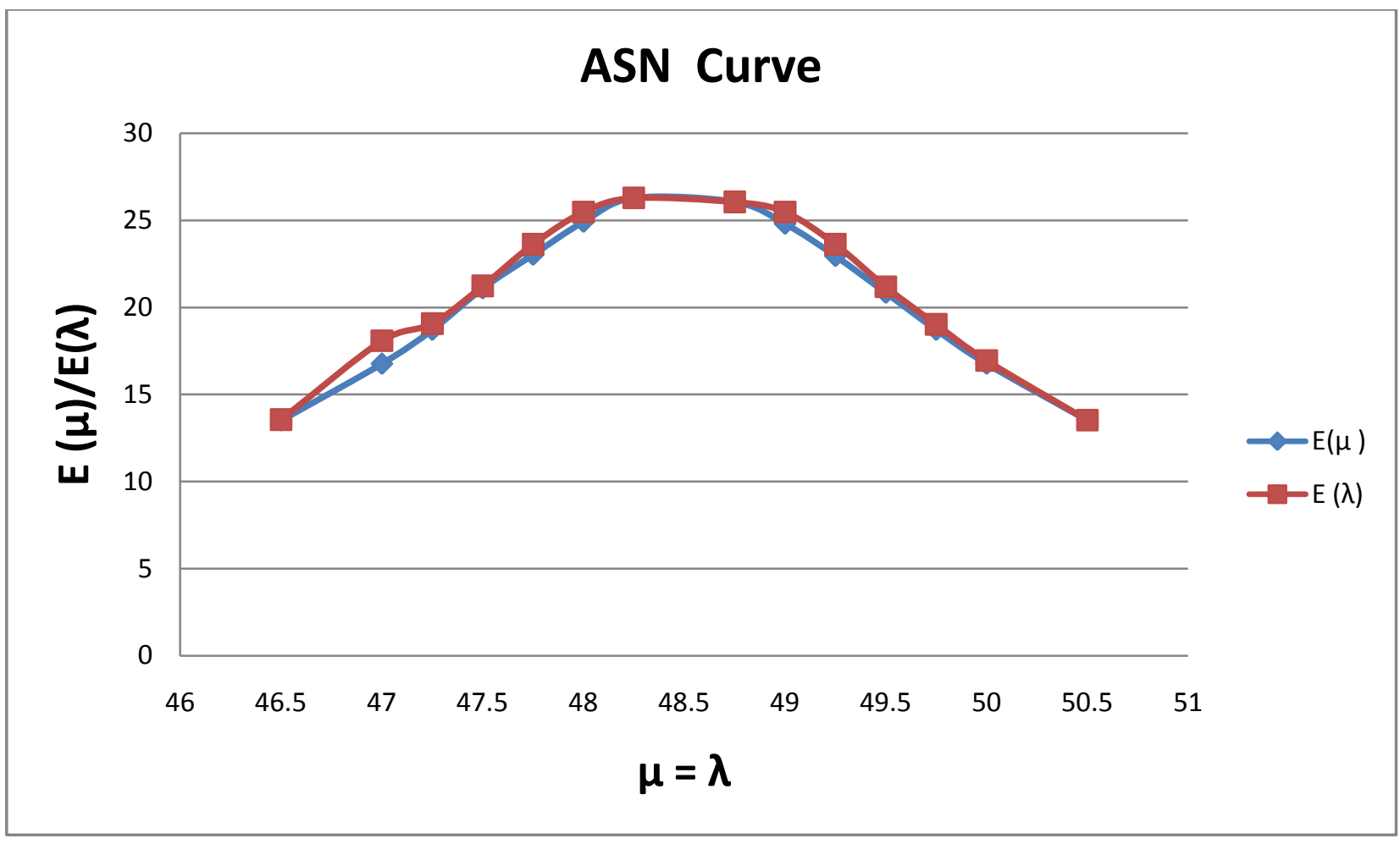

Fig: 2 ASN Curve for SNTP 


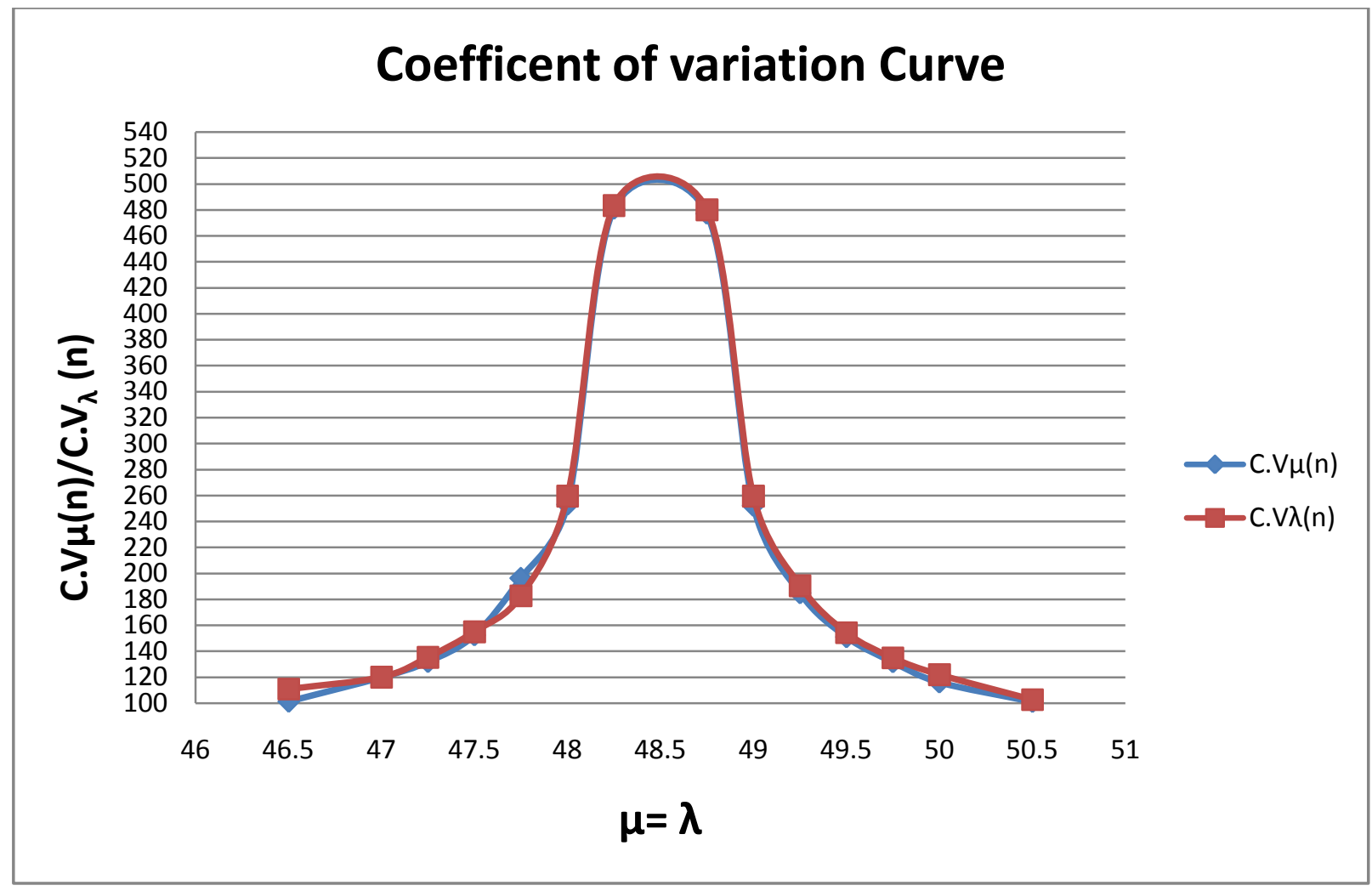

Fig:3 Coefficent of variation curve for SNTP

\section{CONCLUSION}

However, the criterion suffers from the following limitations.

(a) In general, no uniformly test exist depending on $E_{\mu}(n)$

(b) ASN function, $\boldsymbol{E}_{\mu}(\boldsymbol{n})$ alone in unable to reflect the consistent behaviour [closeness between $\mathrm{n}$ and $\boldsymbol{E}_{\mu}(\boldsymbol{n})$ ] of $\mathrm{n}$ as we updating ' $\mu$ ' in view of prior variation.

To overcome these limitations, a suitable measure of relative dispersion called $C V_{\mu}(n)$ is used to analyze this consistency (closeness) between $\mathrm{n}$ and $\boldsymbol{E}_{\mu}(\boldsymbol{n})$. Based on this criterion, an admissible sequential test of strength ( $\alpha$, $\beta$ ) with maximum consistency is called a desirable test.

In the light of above developments, we are able to conclude the following important results when posterior has been updated on using experimental data,

(i) SNTP are non-robust in respect of $\alpha, \beta, E_{\mu}(n)$ and when $\mu$ is considered as a random variable. In the present data setup and from fig. (2), we observe that $E_{\lambda}(n)$ tends to be uniformly higher than $E_{\mu}(n)$ for all varying values of $\mu=$ $\mathrm{E}(\mu)=\lambda$. However, the random variable $\mathrm{n}$ tend to be uniformly less consistent as $\boldsymbol{C} \cdot \boldsymbol{V} \cdot \lambda(\boldsymbol{n})$ is uniformly higher than $C V_{\mu}(n)$ for values of $\mu=\mathrm{E}(\mu)=\lambda<47$. On the other hand, the random variable $\mathrm{n}$ tend to be uniformly more consistent as $C \cdot V \cdot \lambda(n)$ is uniformly less than $C V_{\mu}(n)$ for values of $\mu=E(\mu)=\lambda>50$ (ii) A comparison of the two OC curves, $\boldsymbol{L}(\boldsymbol{\mu})$ and $\mathrm{L}(\lambda)$, in fig.(1) reveals that $\alpha$ and $\beta$ both tend to be equal for all values of $\mu=\mathrm{E}(\mu)=\lambda$.

However the values of $\mu$ and $\lambda$ around the values $\mu=\lambda=47$ or 50 , the SNTP are observed to be equally consistent or robust.

The conclusion is that SNTP are found to be non robust in respect of $\alpha, \beta, A S N$ and consistent behaviour of $n$, when $\mu$ is considered as a random variable. Therefore, SNTP be used cautiously whenever there is a concern about the random variations in $\mu$.

\section{REFERENCES}

[1] Wald, A. (1947), “ sequential analysis", John Wily and sons, New York, USA.

[2] Montage, E.R. and Singpurwalla, N.D.(1985), "Robustness of sequential exponential life testing procedures", JASA,80,715-719.

[3] Sharma K.K. and Rana, R.S.(1990), "Robustness of sequential Gamma life testing procedure", Micro electron Reliab., Vol.30 (6),1145-1153.

[4] Sharma K.K. and Rana,R.S.(1991), "Robustness of sequential Gamma life testing procedure in respect of expected failure times", Micro electron Reliab., Vol. 31(6), 1073-1076.

[5] Sharma K.K. Singh B. and Goel J.(2009)," Sensitivity Analysis of Sequential Normal Testing Procedure."J.Stat. \& Appl. Vol.4, No.1, 45-57.

[6] Bazovsky, I.(1961), "Reliability theory and practice", Practice Hall, New Jersey. 
[7] Box, G.E.P. and Tiao(1973),"Bayesian inference in statistical analysis".

[8] Davis D.J.(1952), "The analysis of some failure data", J. Amer. Statist. Assoc.,47, 113-150.

[9] Fryer and Holt(1970), "On the robustness of the standard estimate of the exponential mean to contamination", Biometric, 57, 641-648.

[10] Fryer and Holt(1976),"On the robustness of the power function of the one sample test for the negative exponential distribution", Commun. Statistics, A5, 723734.
[11] Harter, L. and Moore, A.H.(1976), "An evaluation of exponential and Weibull test plans", IEEE transactions on Reliability, 25, 100-104.

[12] James E.Hall(1979), "Minimum Variance and VOQL Sampling Plan”, Techno metrics, Vol.21, No.4, 555-565.

[13] Johnson, N.L., and Kortz, S.(1969): Discrete Distributions, John Wiley and Sons, New York.

[14] Martz, H.F. and Waller, R.A.(1982), "Bayesian Reliability Analysis", John Wiley and Sons, New York, USA.

[15] Zellen and Dannemiller(1961), "The Robustness of the life testing procedure derived from the exponential distribution", Techno metrics, 3, 29-49. 